\title{
RADIO GALAXIES AND QUASARS, I
}

\author{
Invited Discourse C given on August 28, 1967 in the Lucerna Hall
}

\section{RYLE}

(University of Cambridge, England)

If we observe the sky with a radio telescope operating at metre wavelengths, we find first a continuous background mainly due to the Milky Way - which we observe undimmed by the obscuration which spoils our optical view. In addition we find compact sources, a few minutes of arc or less in extent. About 8000 of these compact sources have now been discovered using large instruments, but only a few hundred of the most intense have been studied in any detail; a few are within our Galaxy and represent the emission from the remnants of supernova explosions, but many of them are found to be associated with faint galaxies. From their distances we can conclude that their radio emission is very great, in some cases more than a million times greater

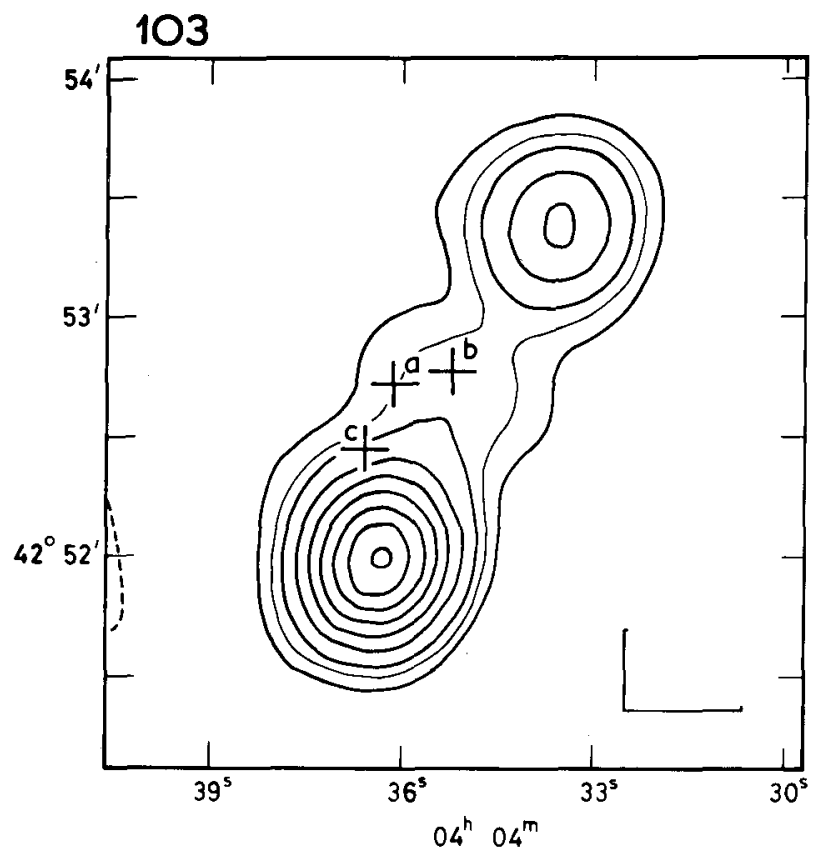

FIG. 1. 3C 103 consists of two compact components separated by 88" arc; the cross (a) represents the position of a $16^{m} D$ galaxy with which the radio source is probably related.

Perek (ed.), Highlights of Astronomy, 33-44. (1) I.U.A. 
than that from our own Galaxy or the Andromeda nebula. These powerful sources are known as 'radio galaxies'.

Observations with instruments of high resolving power show that about $60 \%$ have a double structure, with radio emission from two components, one on each side of the related galaxy; the two components are frequently of unequal intensity, and they may be located at unequal distances from the galaxy.

Their appearance and other evidence, which will be discussed by Allan Sandage, suggest that the radio-emitting material has been ejected by an explosion within the galaxy.

The maps shown in Figures 1-4 were made with the one-mile telescope at Cambridge - which has a resolution of about $25 \mathrm{sec}$ of arc when operating at a wavelength of $21 \mathrm{~cm}$.

Some sources show a more complex structure, in which a number of components can be distinguished, sometimes linked with bridges of emission (Figures 2, 3, 4). Such sources might be the result of a series of lesser explosions.

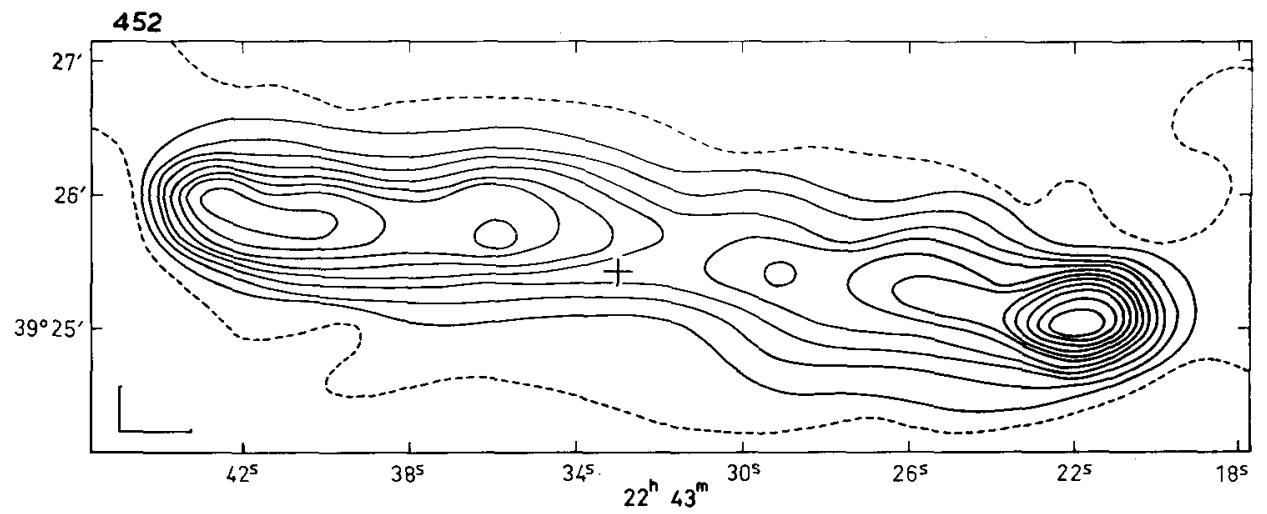

FIG. 2. $3 C 452$ contains a number of peaks in a long ridge of emission, suggesting that more than one release of energy has occurred. The cross marks the position of a $16^{m} E D$ galaxy, which may be the source of the energy.

In addition to these sources, some $35 \%$ are of much smaller angular size; observations with long-baseline interferometers, particularly that between Jodrell Bank and Malvern in England, and by observations of lunar occultations notably at Parkes in Australia and Arecibo in Puerto Rico, have shown that many of them are less than $1^{\prime \prime}$ arc in extent; in some cases the structure is again double, with components $\sim 0 \cdot 1^{\prime \prime}$ arc in diameter, separated by a few seconds of arc.

Many of these compact sources have been found to be associated with very small optical objects which look like stars, and they have become known as quasi-stellar sources (QSS). Dr. Sandage will describe how their optical spectra contain lines which can only be interpreted if very large red-shifts are adopted. The origin of these red- 


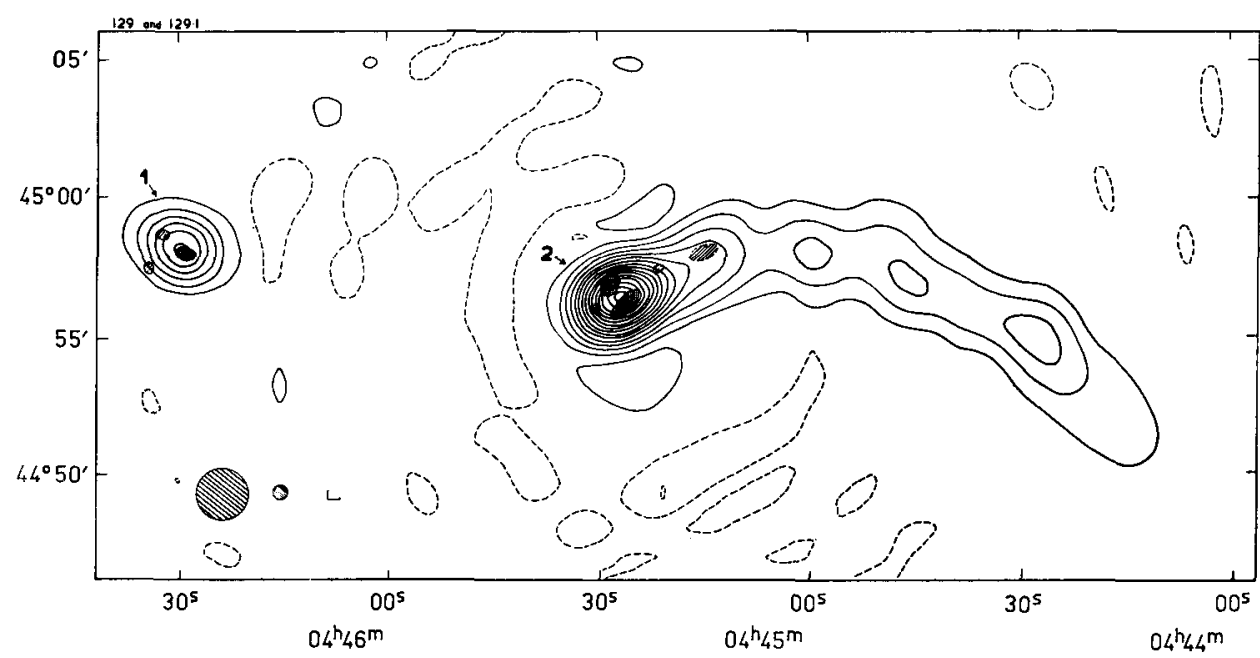

FIG. 3. 3C 129 and 129.1 are probably related, and each contains a number of compact components as indicated by the shaded areas which were observed at a wavelength of $21 \mathrm{~cm}$. The general distribution is shown by the contours observed at a wavelength of $75 \mathrm{~cm}$. The resolution of the instrument at the two wavelengths is indicated by the shaded circles in the lower corner of the map. - The curved distribution of 3 C 129 (source number 2) suggests that a series of explosions may have occurred during the rotation of the parent body.

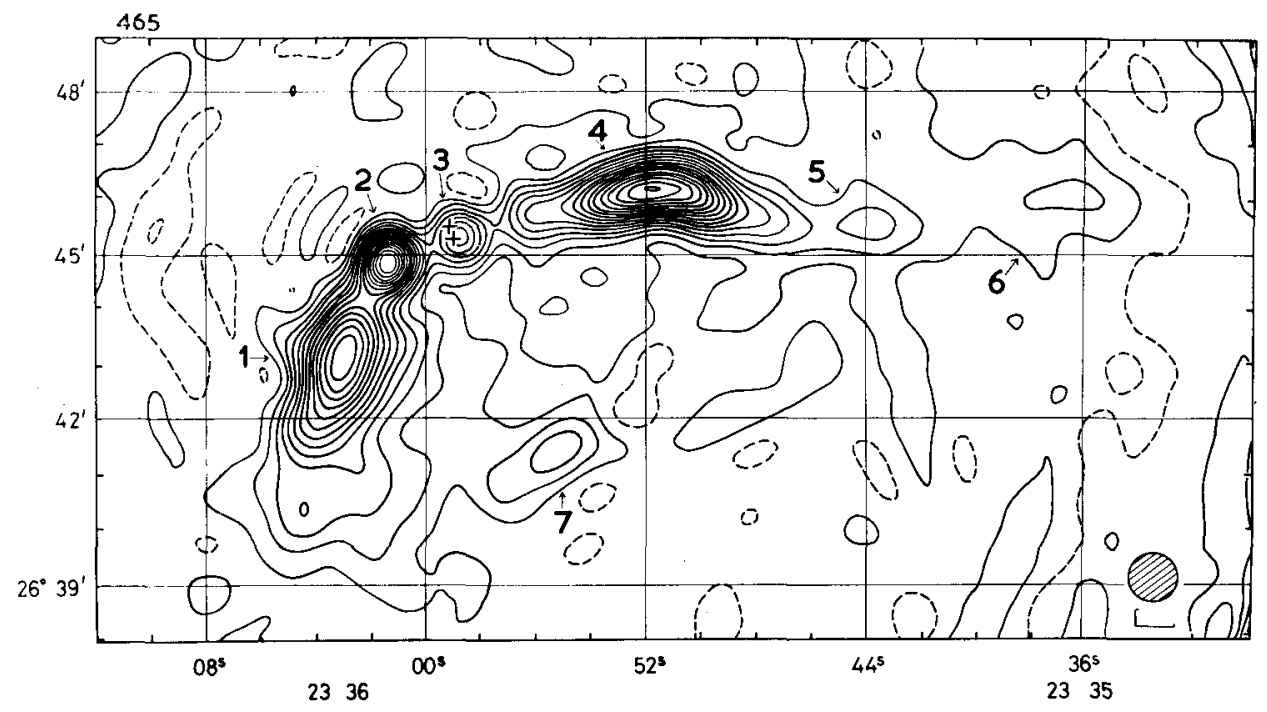

FIG. 4. 3C 465 comprises seven components; the most compact (3) is unresolved and coincides with the brighter of the two nuclei of NGC 7720. It seems probable that a series of explosions within NGC 7720 was responsible for all the components. 
shifts is still controversial; the most obvious interpretation is that they are Doppler shifts due to the general expansion of the Universe, and that the QSS are at great distances. In order to avoid the necessity for objects having such very great radio, optical and UV emission, and to explain some of the peculiarities of the observed red-shifts, some astronomers believe that they are comparatively local - though still extra-galactic. In this case some other explanation must be sought for the red-shifts. Allan Sandage will discuss these two alternatives in detail, but I shall be presenting evidence which supports the former interpretation, that they are at great distances.

Observationally we can distinguish QSS from radio galaxies in a number of ways, although it is very difficult to draw a sharp dividing line; many sources show characteristics belonging to both classes of object.

(a) QSS show fluctuations of radio intensity with periods of $\sim 1 \mathrm{sec}$, due to diffraction in the interplanetary medium; this effect, which is analogous to optical 'seeing' only occurs for sources having an angular diameter $<1$ " arc, and is proving valuable as an additional method of investigating their angular structure in the range $1^{\prime \prime}$ to 0.01 arc.

Here we should note that $3 \mathrm{C} 295$, which is associated with a massive galaxy, and has a compact double structure, also scintillates, revealing the presence of at least one very small component. On the other hand, $3 \mathrm{C} 47$ and 263 , which are both identified with optical QSS, are extensive double radio sources. 3C 225 and 267 are also extensive double sources, but each contains one very compact component as revealed by interplanetary scintillation; neither has a conclusive optical identification.

(b) Observations at short wavelengths $(<10 \mathrm{~cm})$ have revealed longer-term variations, of some months, which cannot be explained in terms of diffraction in the interplanetary, interstellar or intergalactic media, and which must be attributed to intrinsic variations of the source itself.

The existence of such variations indicates an upper limit to the physical dimensions

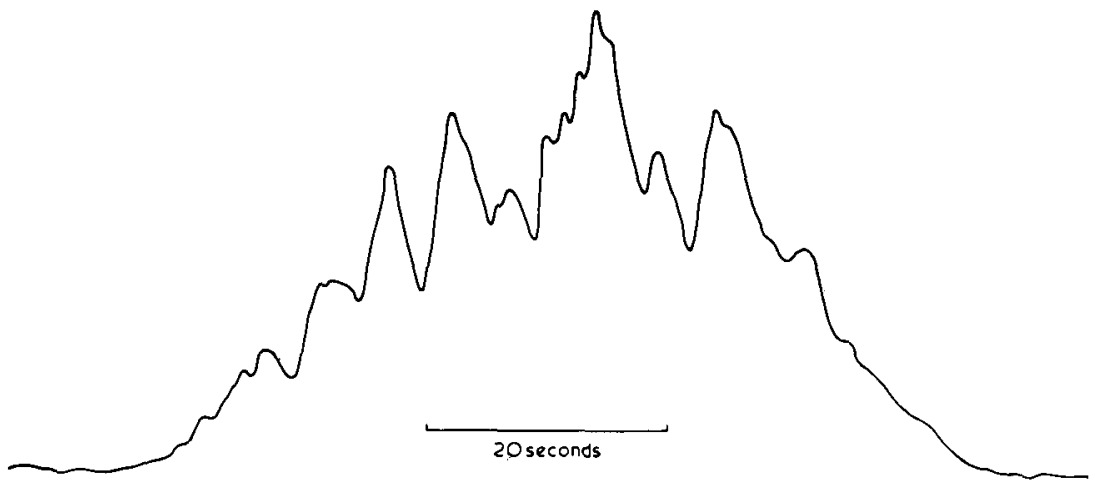

FIG. 5. Record showing the fluctuations in the intensity of $3 C 48$ due to diffraction in the interplanetary medium. The observations were made with a transit instrument. 


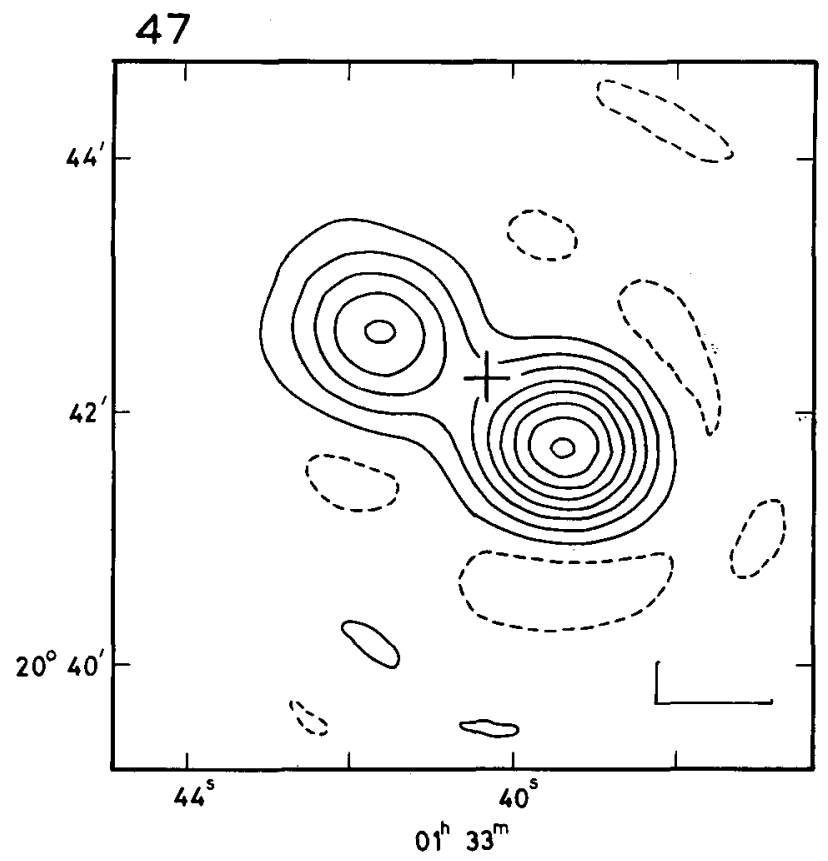

FIG. 6. $3 C 47$ consists of two components either side of an optical $Q S S$; if the red-shift of 0.425 is interpreted as of cosmological origin their physical separation is $208 \mathrm{kpc}$, a figure similar to that of many radio galaxies.

of the source of a few light months. There are in fact even more rapid variations in the optical emission, as Dr. Sandage will discuss.

Here again, it has been found that the radio source associated with the galaxy NGC 1275 also shows fluctuations at high frequencies, and shows that very compact radio components certainly exist within some galaxies.

(c) Figure 8 shows a third way of distinguishing QSS by their radio spectra; most radio galaxies have a spectrum which may be described by an exponential, but QSS normally show a marked cut-off at low frequencies, as shown in Figure 8.

In some cases this cut-off occurs at quite a high frequency, and it is these sources which are found to be the most compact as shown by their variability; this result suggests that the cut-off should be attributed to self-absorption effects within the source itself, and also indicates why variability is not observed at low frequencies.

The physical explanation for this cut-off is now reasonably well understood. The only satisfactory mechanism which has been suggested to account for the generation of radio waves in both QSS and radio galaxies is the synchrotron process, in which electrons of high energy are accelerated in a magnetic field. This mechanism not only provides a simple explanation for the observed spectra - including the low-frequency cut-off in the most compact sources - but also predicts the presence of linear polariza- 


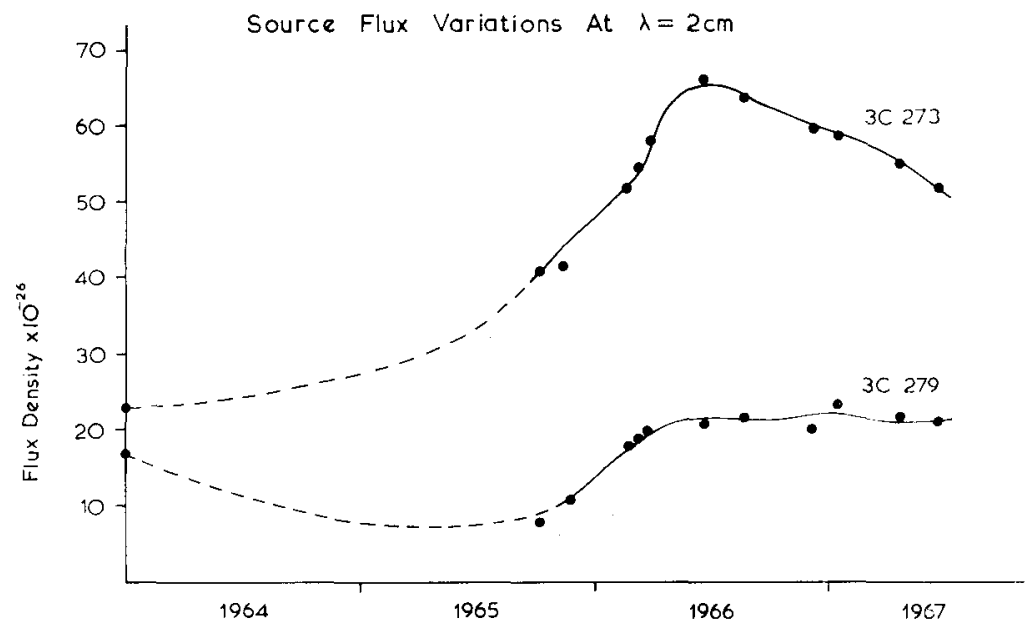

FIG. 7. Observations made at Greenbank of the emission from $3 C 273$ and 279 at a wavelength of $2 \mathrm{~cm}$ show marked variations of intensity.

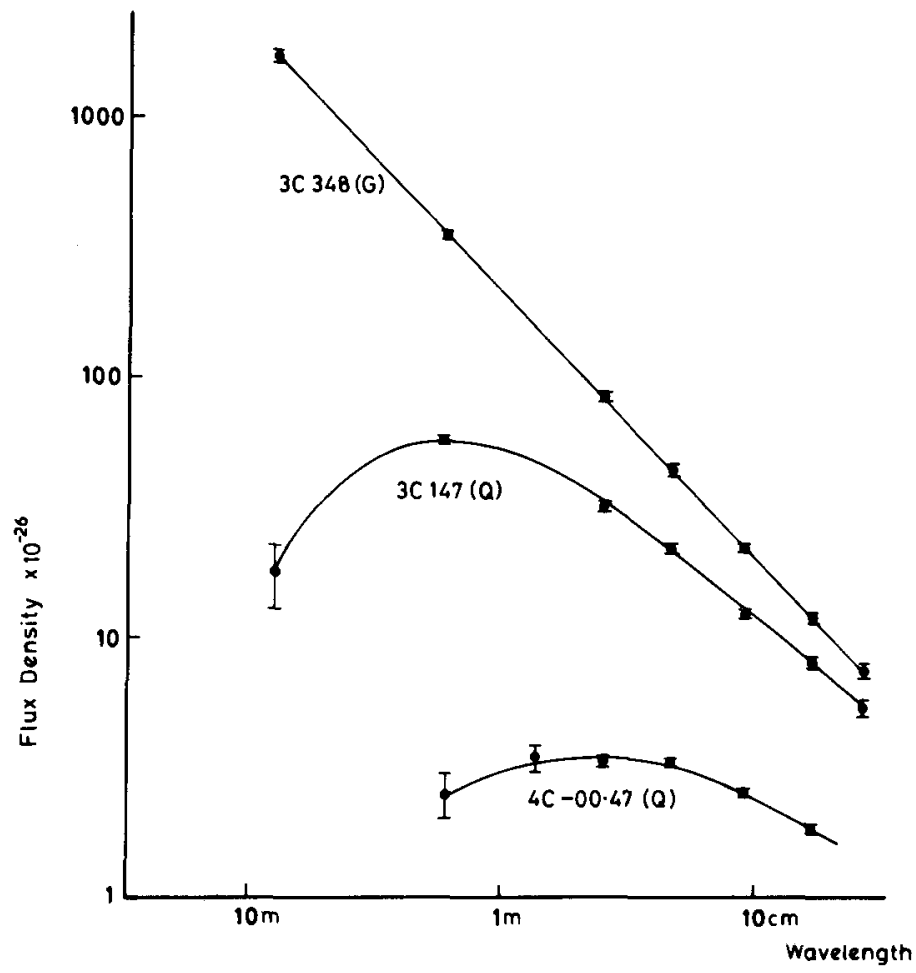

FIG. 8. The radio spectra of a radio galaxy (3C 348) and two QSS (3C 147 and 4C-00.47). 
tion in the emission from sources where the geometry of the magnetic field is simple; the observation of such polarization in many sources has provided further confirmation of the synchrotron mechanism.

For sources with a low-frequency cut-off whose angular extent and distance are known, we can derive the magnetic field and the number and energy distribution of the high-energy electrons, so that the total energy required to account for the radiation may be found. In other cases where no cut-off in the observed spectrum occurs it is still possible to establish a lower limit to the energy in the particles and magnetic field. Estimates of the rate at which the energy will be lost may also be made so that the life time of different types of source may be estimated.

The energy which is necessary to account for the most powerful radio galaxies which must presumably be released within the parent galaxy - is of the order of $10^{61}$ ergs, equivalent to the conversion to helium of a mass of hydrogen of about $10^{9} M_{\odot}$. It is significant that if the red-shift of the QSS is interpreted as of cosmological origin, the emission from the most powerful of these sources also implies energies of the same order.

Even in the extensive radio galaxies the lifetime of the electrons is only about a million years, and the large separation of the emitting regions from the parent galaxy (of $100 \mathrm{kpc}$ or more) implies that they must have been ejected from the galaxy with velocities comparable with the velocity of light.

These results suggest that QSS and radio galaxies may simply be different stages in the evolution of the same class of source. It seems certain that radio galaxies must have their origin within the related galaxy and that the source must therefore have passed through a more compact stage; can we indeed release $10^{61}$ ergs within a time $<10^{6}$ years in a galaxy and not observe it by optical or radio means? It is equally certain that the energy density in QSS is so high that they must expand very rapidly; where are the sources resulting from such an expansion if they are not the radio galaxies?

It is suggestive that the recent high-resolution observations(Jodrell Bank-Malvern) of a number of the most compact QSS known, also reveal a double structure, so that if we adopt the cosmological interpretation of their red-shifts, two-component sources are now known which cover a range of separations from $1-450 \mathrm{kpc}$.

A number of double sources from the $3 \mathrm{C}$ catalogue are indicated in Figure 9, together with the physical size and intrinsic radio power derived on the assumption that the red shift is cosmological. The nature of the related optical object is also shown.

If the red-shifts of the QSS are not cosmological, the similarity in the derived powers would be a remarkable coincidence. The impossibility of making any clear division between QSS and radio galaxies suggests strongly that the two classes of source are related.

In order to visualize how QSS and radio galaxies might evolve, a simple model may be constructed which supposes that a large amount of energy $\left(\sim 10^{61} \mathrm{ergs}\right)$ is suddenly released at the centre of a galaxy, and that this gives rise to the ejection of two plasma 


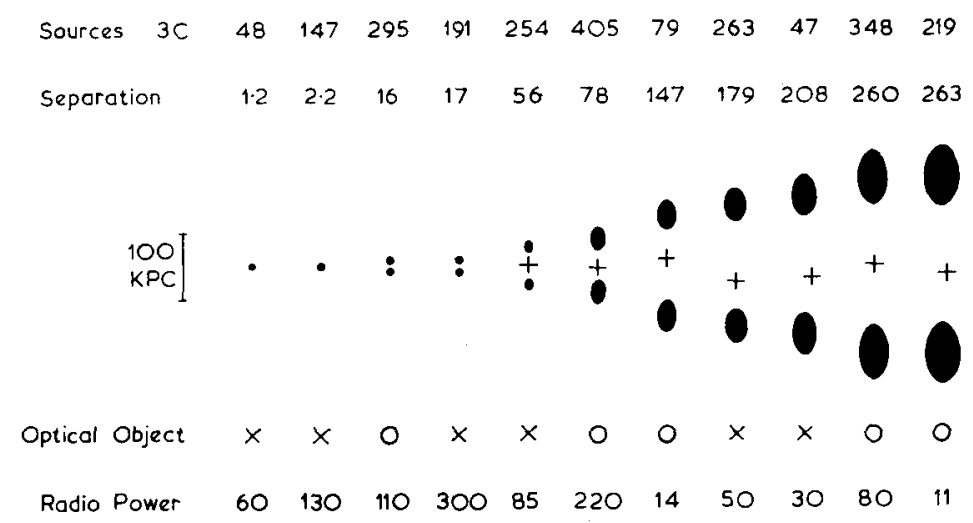

FIG. 9. The physical extent ( $\mathrm{kpc}$ ) and radio power (in units of $10^{25}$ watts ster ${ }^{-1} \mathrm{~Hz}^{-1}$ ) of a number of double radio sources, together with the nature of the related optical object ( + QSS; O Galaxy).

clouds travelling out in opposite directions with an initial velocity $\sim c$. Except in the case where the ejection has occurred along a line perpendicular to the line of sight the components will be observed at significantly different ages because of their relativistic velocities, and they will also not be separated by equal angles from the galaxy.

By using the observed angular separations of the components from the optical object in a number of actual sources, the velocity of ejection and ages of the individual components may be found, so that for each source the variation of emission with time may be compared on the basis of the model.

Less accurate data may be obtained in a similar way for the more compact sources. It is seen that the observations of a large number of sources are in reasonable agreement with a model in which the emission at short wavelengths remains constant for $\sim 10^{5}$ years and then rapidly decreases to a small value after $\sim 3 \cdot 10^{6}$ years. If a similar curve is drawn for the emission at long wavelengths the effects of self-absorption may be seen to persist until $\sim 3 \cdot 10^{4}$ years.

These results suggest that all powerful extra-galactic radio sources may belong to the same class and that the wide spread in the properties is mainly due to their different ages. Sources showing the characteristics of both QSS and radio galaxies occur within, and only within, the age limits $3 \cdot 10^{4}-3 \cdot 10^{5}$ years. The rapid decrease in emissivity after $\sim 10^{5}$ years corresponds approximately to the age at which the plasma clouds pass out of the galaxy, and agrees with the expected decrease of emission from a plasma cloud expanding into a vacuum.

Many suggestions have been made to account for the large amount of energy needed, including the possibility of a chain reaction of supernovae, mechanisms occurring during star formation, the annihilation of matter and anti-matter, and the gravitational collapse of a supermassive star. Some support for the first of these hypotheses has been provided by some recent observations of the Seyfert galaxy NGC 1275, which gives the impression of a QSS in slow motion. 


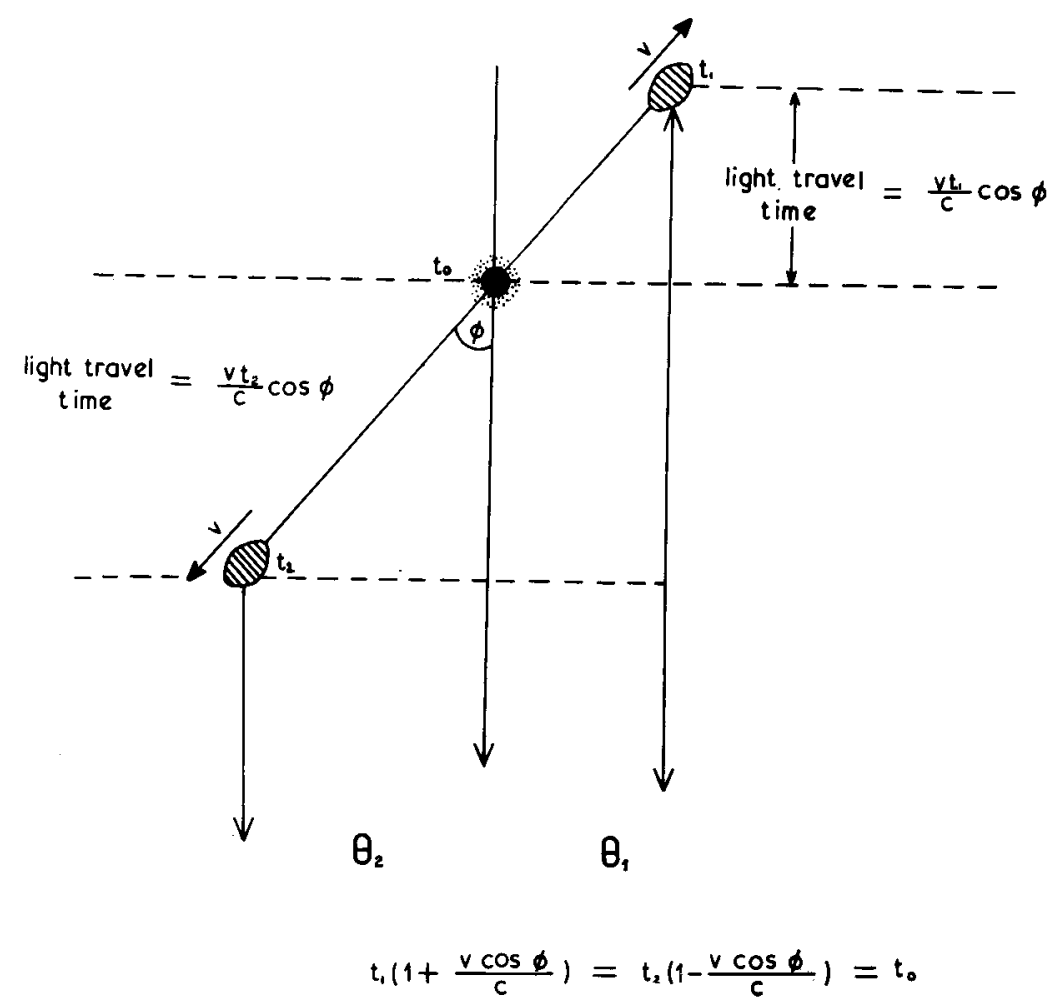

FIG. 10. The ejection of two plasma clouds in opposite directions from a galaxy at relativistic velocities will give rise to a double source whose components will be observed at different proper ages.

The nucleus of the galaxy contains a compact radio source, variable in periods of a few years, with a low-frequency cut-off of $5000 \mathrm{MHz}$; it is thus similar, though of much less power, to some of the most compact QSS. This very compact source could be accounted for by a large supernova event comparable with that which has produced the intense galactic radio source Cassiopeia $A$. The radio emission from the nucleus as a whole also shows a low-frequency cut-off $(400 \mathrm{MHz})$ and could be maintained by the occurrence within the nucleus of one such supernova every few years. The optical evidence supports the idea that there has been a succession of supernovae within the nucleus.

Recent observations have revealed radio emission from more extensive components, including the distorted halos of two neighbouring galaxies within the Perseus Cluster which can only be accounted for in terms of particles ejected from NGC 1275. A rate of production of particles at least as great as that occurring at the present time must therefore have persisted for 5-10 million years, a result confirmed by the observation by Margaret Burbidge of gas streams in the outer parts of NGC 1275. The total energy released is therefore comparable with that of the most powerful sources known, 


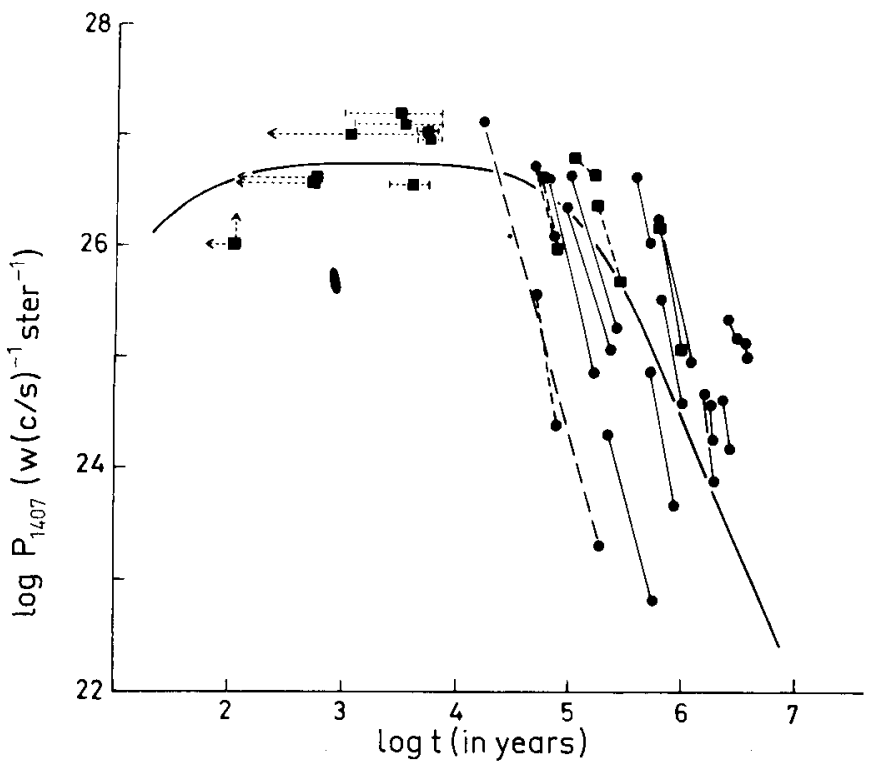

FIG. 11. The emission from each component of a number of double sources plotted against the component age, as derived on the basis of the simple source model.

but it has been released over a period of several million years. The possibility of such a supernova process occurring very rapidly, perhaps by a chain reaction as suggested by Geoffrey Burbidge, which would allow all the energy to be released within the light travel time across the nucleus, might therefore provide the energy source for QSS and radio galaxies.

Whatever their detailed nature, the existence of intrinsically very powerful radio sources is of great importance in testing cosmological models; with large radio telescopes they can be detected at great distances and hence should provide information on the structure of the Universe at earlier epochs than can be reached with optical telescopes.

The most important of these cosmological tests are of three kinds:

(i) Determination of the numbers of sources in different ranges of flux density;

(ii) Examination of the isotropy of the sources;

(iii) Determination of the contribution to the background radiation of sources below the limit of detection.

The results obtained at Cambridge in the $4 \mathrm{C}$ survey and at Parkes have shown a distribution of sources which is isotropic at flux densities which correspond to values of the red-shift $z$ of at least 1 , but their distribution is not uniform in depth; with decreasing flux density the number of sources initially increases much faster than expected for a uniform population, suggesting that at earlier epochs either the number or the intrinsic power of radio sources was greater than at present. This increase has 
been attributed to QSS alone but there are important observational selection effects which make it less likely that faint radio galaxies will be identified than QSS; the greater intrinsic optical luminosity of QSS and the difficulty of defining the position of a galaxy associated with an extensive double source are particularly important. It seems probable that all powerful radio sources exhibit the effect, and that it can only be explained in terms of a cosmological model which permits evolutionary changes.

The greatest excess of sources appears to be due to sources having red-shifts $z \sim 2-3$.

New observations with the one-mile telescope at Cambridge have allowed the detection of sources some 100 times fainter than the limit of the $4 \mathrm{C}$ and Parkes surveys. The results are shown in Figure 12, in which the number of sources $(N)$ observed in unit solid angle having a flux density greater than $S$, is compared with $N_{0}$, the number expected on a static Euclidean universe. The variation of $N / N_{0}$ with $S$ for an Einsteinde Sitter model is also shown.

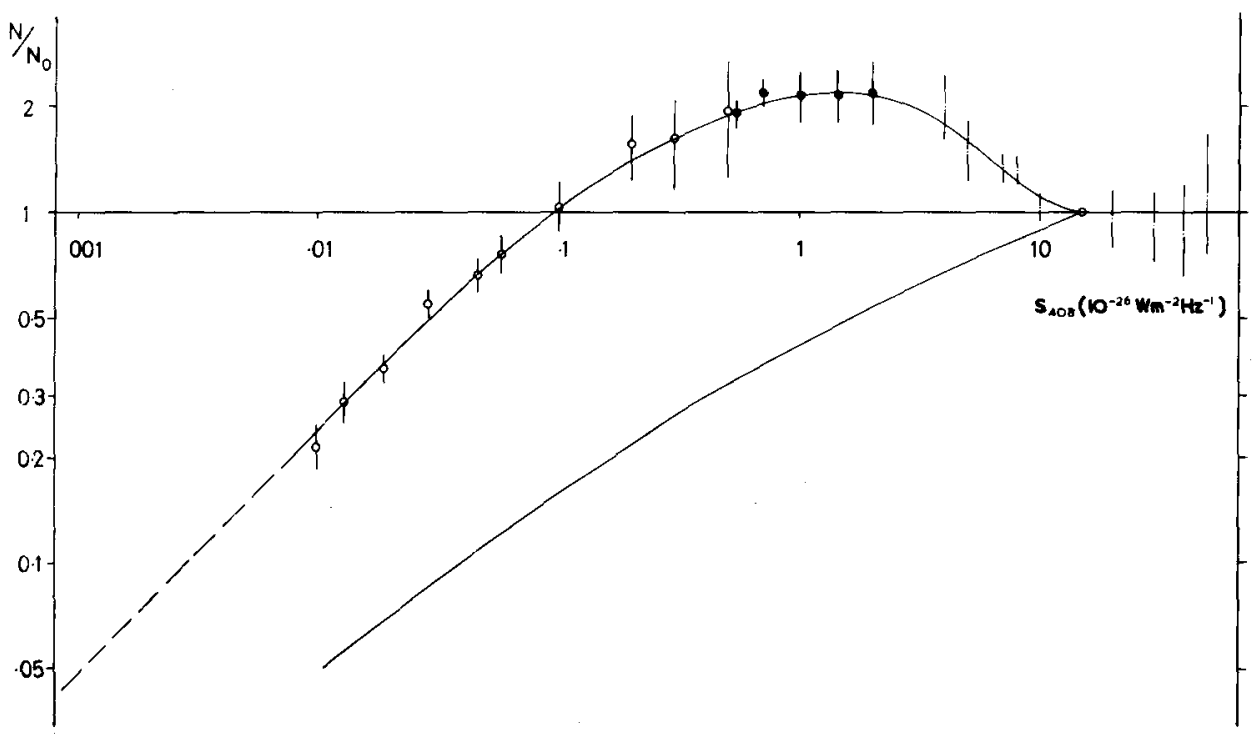

FIG. 12. Plot of the ratio of the number of sources $(N)$ per unit solid angle having a flux density greater than $S$, to the number (No) which would occur in a static Euclidean universe. The lower line represents the curve for an Einstein-De Sitter model. - The extrapolated dotted curve would account for effectively the whole of the integrated extragalactic emission.

These results show that at still smaller flux densities, the excess of sources which is so marked at $S \sim 10^{-26} w \cdot m^{-2} \mathrm{~Hz}^{-1}$ is followed by a remarkable convergence in the number of sources. This result indicates that a cut-off in the number of sources must occur at red-shifts $z \sim 3$. Since any cut-off will be blurred by the spread in the intrinsic radio luminosity, the relatively rapid convergence observed implies that this cut-off must be very sudden. 
The existence of this cut-off is confirmed by considering the integrated emission from extra-galactic sources; the contribution from the sources which have now been observed individually is about half that of the total integrated emission, and it is evident that most of the remainder must be associated with sources of smaller intrinsic power at smaller red-shıfts.

These observations provide strong additional evidence against any explanation of the source counts in terms of a local non-uniformity in the distribution of radio galaxies or QSS; the observed isotropy implies that we would have to be situated very close to the centre of such a local system, and since the observed sources account for most of the extra-galactic background emission, there can be few other such systems within $z \sim 1$. We can therefore only accept a local origin if we suppose that we are situated in a specially favoured place in the Universe - a situation which has been distasteful to astronomers since the time of Copernicus.

It appears that the source counts and the integrated extra-galactic radio emission reveal important evolutionary effects associated with the expansion of the Universe; prior to some epoch corresponding to a red-shift $z \sim 3$ (which may be related to the formation of galaxies) radio sources apparently did not exist. Subsequently, galaxy formation may have led to the birth of radio sources which were either more powerful or more numerous than they are at the present epoch.

Entirely independent evidence for an evolutionary cosmology has been provided by the recent discovery of isotropic microwave background radiation having a blackbody spectrum; the only explanation which has been proposed to account for this emission is that it represents the fossil radiation from the 'fireball' associated with the highly condensed initial stages in evolutionary cosmologies.

I have tried to summarize the radio evidence on radio galaxies and QSS, but much of our information on these strange objects has of course been provided by optical observations. Dr. Allan Sandage will continue with this side of the story. 